\title{
L'Impero romano d'Occidente e i Barbari: le invasioni e la disfatta economica del V secolo d.C.
}

\author{
Alberto Giudice
}

\section{Premessa}

Gli approfonditi studi che si sono svolti in questi ultimi decenni sul tardo antico hanno consentito di precisare le caratteristiche del periodo e di generare un acceso dibattito che, ancora oggi, dilania il mondo accademico che risulta essere smembrato in due scuole di pensiero: continuisti e catastrofisti. ${ }^{1}$ I primi, a seguito della rivoluzione copernicana di Peter Brown ${ }^{2}$, considerano il tardo antico come un'età di transizione che segna il graduale passaggio dall'epoca antica a quella medievale ${ }^{3}$. I secondi, al contrario, giudicano il periodo come un'epoca di cesura e di rottura con il mondo antico ${ }^{4}$.

Appare evidente che la diversa valutazione che si da all'epoca ha portato ad una distinta considerazione delle invasioni barbariche. Secondo i continuisti, infatti, le popolazioni che attraversano il confine germanico non provocano alterazioni nella struttura economica e politica dell'impero romano d'Occidente e affermano che i barbari si sono semplicemente "accomodati" nel territorio di Romas. Secondo i catastrofisti, invece, gli invasori hanno causato il declino economico e sociale di Roma portandola verso la caduta sancita nel 476 d.C. ${ }^{6}$

La sterile controversia, che ancora oggi è attiva nel mondo accademico, non ha consentito di precisare come i Barbari abbiano alterato il sistema economico romano poiché le due fazioni non hanno analizzato come gli “invasori” si siano inseriti all'interno di un apparato consolidato ma fragile.

Il modello che qui si propone è sostanzialmente diverso da quello tradizionale: non si parlerà di continuità o di rottura ma di una alterazione sostanziale che porterà alla nascita di un nuovo mondo economico fondato su nuove basi.

Per raggiungere questo scopo si sono analizzate sia le fonti letterarie che quelle archeologiche in maniera paritetica: nessuna delle due ha prevalso sull'altra in modo da non far risaltare ne l'idea della continuità ne quella della rottura.

\footnotetext{
${ }^{1}$ Sul dibattito storiografico si vedano gli approfonditi studi di GIARDINA 1999, pp. 157-180; MARCONE 2004, pp. 25-36; Idem 2007, pp. 267-280; DE GIOVANNi 2007, pp. 1 - 38, DEMANDT 2007, pp. 578608.

${ }^{2}$ Lo studioso si sofferma sull'analisi delle modifiche sociali e religiose che si verificano nel corso del periodo che analizza ma non sui problemi economici che dovette affrontare l'Impero romano d’Occidente durante gli ultimi secoli di sopravvivenza. Secondo lo storico irlandese nel tardo antico non si verificò un cambio radicale della società: il vescovo si sostituisce al senatore, continuano gli antichi legami di fedeltà e la cultura classica continua ad essere coltivata; a seguito dell'arrivo dei Barbari l'impero aveva «conservato tutto: tutto tranne un imperatore» BROWN 1974, pag. 106; Idem 2001.

${ }^{3}$ Cracco RugGini 1993, pp. XXXIII-XXXVI sgg..; BowERsocK 1996, pp. 29-43; CrACCO RUGGINI 2002, pp. 351-379; BOWERSOCK 2002, pp. 376-379 ; VERA 2002, pp. 349-350; BOWERSOCK 2004, pp. 7-13; CRACCO RugGini 2004, pp. 15-23.

${ }^{4}$ CARANDini 1993, pp. 11-38; SCHIAVONE 1996; Giardina 1999, op. cit.; HeATHer 2008; WARDPERKINS 2008.

${ }^{5}$ PiRENNE 1937; GOFFART 1980.

${ }^{6}$ Petralìa 1995, pp. 38-87; HeAther 2008, op. cit.; WARD-PERKINS 2008, op. cit.
} 


\section{Le fonti letterarie}

La divisione dell'impero romano in due compagini statali ebbe notevoli ripercussioni sulla parte occidentale che subì, nei primi decenni del V secolo, una serie di invasioni che provocarono il graduale declino della struttura sociale e politica: a partire dal 401402 l'Italia patì l'invasione di Alarico e i Goti a cui seguì, nel 405-406, quella di Radagaiso e di Alarico dal 408 al $412^{7}$.

Anche la Gallia dovette affrontare numerose invasioni: nel 407-409 Vandali, Svevi e Alani varcarono, approfittando della chiamata delle truppe confinarie in Italia per fermare Radagaiso, il confine romano. A seguito della conquista di parte della Gallia i Vandali varcarono i Pirenei e conquistarono la penisola iberica ${ }^{8}$.

Queste incursioni provocarono grosso sgomento tra la popolazione romana come si evince dalla lettura delle fonti letterarie. Esemplare, a questo proposito, è la fonte di Eugippio che, nella Vita di Severino, descrive la condizione del Norico Ripense:

«Finché esistette l'impero romano, i soldati di molte città vennero mantenuti a pubbliche spese per difendere la frontiera. Quando tale pratica cadde in disuso, queste truppe scomparvero insieme alla frontiera.» ${ }^{9}$

La fonte, descrivendo lo stato in cui versava la provincia nella seconda metà del V secolo d.C., presenta il completo abbandono di questo territorio da parte dell'esercito imperiale. Sempre Eugippio narra un evento che consente di comprendere cosa provocarono le invasioni barbariche. A detta dell'agiografo esisteva soltanto una guarnigione imperiale che prestava servizio nella città di Batavis. Per ricevere la paga, tuttavia, i soldati dovevano inviare in Italia un'ambasceria che la riscuoteva. Ma non fece più ritorno poiché «furono uccisi dai Barbari durante il viaggio» ${ }^{10}$.

Ancora più importante risulta essere un poemetto che, contemporaneo all'entrata dei Vandali in Gallia nel 407, descrive le invasioni:

«Alcuni giacquero in pasto ai cani; a molti la casa in fiamme tolse la vita e fornì il rogo.

In tutti i villaggi e le ville, in campagna e al mercato, in tutte le regioni e le strade, nei luoghi più diversi, c'erano Morte, dolore, Distruzione, Fiamme e Lutti. La Gallia intera giaceva su un unico rogo fumante» ${ }^{11}$.

Le fonti che si sono presentate mostrano i territori come luoghi desolati dove tutto è annichilito a seguito dell'arrivo delle popolazioni barbariche e sembrano essere molto simili a quelle che descrivono l'Africa al momento dello sbarco dei Vandali.

Emblematico a questo proposito è Vittore di Vita che narra l'episodio dell'approdo dei Vandali nel 429:

\footnotetext{
${ }^{7}$ MatThews 1975, pp. 284-306.

${ }^{8}$ COURCELle 1948, pp. 58 - 79; CourTOIS 1955, pp. 42 - 51.

${ }^{9}$ Eugippio, Vita di Severino, cap. 20

${ }^{10}$ Eugippio, Vita di Severino, cap. 20

${ }^{11}$ Orienzio di Auch, Commonitorium, vv. 179-184
} 
«Nella loro furia barbarica giunsero a strappare gli infanti dal seno delle madri e a sbattere gli innocenti contro il suolo. Altri li squarciavano in due tenendoli appesi per i piedi a testa in giù» ${ }^{12}$.

Questa descrizione non si discosta molto da quella di Possidio:

«In tutte le regioni della Mauretania essi sfogarono la loro furia con ogni genere di crudeltà e atrocità, devastando tutto quanto potevano coi saccheggi, le stragi, varie torture, gli incendi e altri crimini indescrivibili» ${ }^{13}$.

Importante, inoltre, risultano anche altre fonti che descrivono lo stato in cui versava l'impero romano d'Occidente nella metà del V secolo d.C.. Degno di esempio, a questo proposito, è Salviano che presenta lo stato romano in forte declino:

«Dov'è ora lo splendore, la dignità degli antichi Romani? Essi erano potentissimi, noi siamo senza forze. Erano temuti; ora siamo noi che temiamo. I barbari pagavano loro tributi; ora siamo noi i tributari dei barbari. I nostri nemici ci fanno pagare perfino la luce del giorno, e dobbiamo comprare il diritto alla vita. Oh, le nostre sofferenze! Come siamo caduti in basso! Dobbiamo addirittura ringraziare i barbari per il diritto di riscattarci! Cosa c'è di più miserevole e umiliante! ${ }^{14}$.

Risulta essere infine ancora più interessante la descrizione dell'impero da parte del c.d. "Cronista del 452 d.C.”:

«Lo Stato romano è stato ridotto in una condizione miserevole da questi disordini, giacchè non c'è provincia in cui non si sono stanziati i Barbari»» 15 .

È chiara la netta relazione che viene posta tra le invasioni barbariche e il declino dell'impero romano d'Occidente.

\section{Le testimonianze archeologiche}

I dati archeologici contribuiscono a chiarire meglio ciò che causano le invasioni barbariche sulle strutture economiche e politiche dell'Impero romano d'Occidente. Lo studio della quantità di ceramica sigillata africana, importata da Roma nel corso del V secolo d.C., evidenzia un evidente variazione che invita a riflettere sulle motivazioni. A partire, infatti, dal IV secolo d.C. la sigillata africana costituisce la quasi totalità di ceramica fine da mensa importata da Roma ${ }^{16}$.

Tale trend continua sino alla metà del V secolo d.C. come dimostrano i dati quantitativi di diversi siti presi in considerazione.

I saggi compiuti nel tempio della Magna Mater a Roma mostrano come sino alla metà del V secolo d.C. la ceramica sigillata africana costituisca circa il 70\% delle importazioni: lo stesso discorso vale per le quantità provenienti dagli scavi eseguiti

\footnotetext{
${ }^{12}$ Vittore di Vita, Persecuzione dei Vandali, I.7

${ }^{13}$ Possidio, Vita di Agostino, 28.5

${ }^{14}$ Salviano, Il governo di Dio, VI. 18.98-9

${ }^{15}$ Cronaca del 452, sez. 138, pag. 662

16 Sull'argomento si vedano gli studi di CARANDINI 1986, pp. 9-19; PANELla 1986, pp. 21-23; CARIGNANI - CiOTOLA - PACETTI - PANELla 1986, pp. 27-43; ANSELMINO - ColetTI - FERRANTINI PANELLA 1986, pp. 45-81; CIPRIANO - VOLPE 1986, pp. 89-96; PANELLA 1993, pp. 613-697;
} 
nella Schola Praeconum. Ciò che balza immediatamente agli occhi è l'evidente crasi che si verifica nella seconda metà del V secolo d.C. dove la quantità di ceramica passa a circa il $10 \%{ }^{17}$.

I dati appena menzionati non valgono soltanto per Roma ma anche per altre città dell'impero romano. Emblematici, a tal proposito, sono i risultati degli scavi compiuti nella città di Luni: dal 35\% di ceramica sigillata africana importata si passa al 5\% alla fine del V secolo d.C. ${ }^{18}$.

Il calo delle importazioni, inoltre, si evince anche dalle analisi dei dati quantitativi delle anfore da trasporto. Nel 425 d.C., infatti, le africane e le orientali costituivano, insieme, il $44.1 \%$ delle anfore importate a Roma mentre alla fine del V secolo rappresentano soltanto il 28,3\% ${ }^{19}$.

A questo dato, infine, ne va aggiunto un altro: a partire dal 450 d.C. si verifica un declino nell'importazione di vino non italiano e l'aumento nell'acquisizione di vino proveniente dall'Italia meridionale ${ }^{20}$.

I dati archeologici che sono stati analizzati dunque consentono di dimostrare che si ha uno stretto legame tra le invasioni barbariche e il declino delle importazioni a Roma, argomento sul quale si rifletterà nel prossimo paragrafo.

\section{Le invasioni barbariche e la rottura dell'economia romana}

Lo studio svolto sulle fonti letterarie e sui dati quantitativi relativi alla ceramica permettono di ipotizzare che vi sia un forte rapporto tra le invasioni barbariche e il declino del mondo romano. Questi elementi però non consentono di capire quali possano essere i motivi che portano alla decadenza dell'impero romano d'Occidente.

Le fonti letterarie mostrano che le invasioni barbariche provocano il disfacimento dell'impero: su questo insistono sia le testimonianze di Eugippio che, come si è visto, descrive la condizione del Norico Ripense, sia quella di Salviano che rappresenta l'impero in completo disfacimento a seguito dell'arrivo dei barbari.

È necessario, ora, comprendere perché gli invasori provocarono il disfacimento e il conseguente declino dell'impero romano d'Occidente: causa che può essere spiegata soltanto se si inquadrano le incursioni nel contesto economico di appartenenza.

È da dire, infatti, che i popoli che invasero il territorio di Roma non si "accomodarono", come si legge in Goffart, o furono stanziati dai romani in territori non popolati ${ }^{21}$. Le fonti dimostrano, al contrario, che i barbari occuparono con la forza gran parte dei territori e non ci fu alcun patto sulla divisione delle risorse presenti in essi. Si conoscono, infatti, soltanto due accordi stipulati nel corso della prima metà del V secolo d.C. tra Roma e gli invasori: il primo del 419 che sanciva lo stanziamento dei Visigoti in Aquitania ${ }^{22}$; il secondo del 442 che decretava la cessione dell'Africa settentrionale ai Vandali di Genserico ${ }^{23}$. Per il resto la conquista di altri territori dell'impero furono estorti con la forza come si evince dalla conquista della Spagna da parte dei Visigoti e dall'occupazione della stessa Africa che fu soltanto sancita dal

\footnotetext{
${ }^{17}$ CARIGNANi - CiOTOLA - PACETTI - PANELla 1986, op. cit.

${ }^{18}$ CIPRIANO - VOLPE 1986, op. cit.

${ }^{19}$ CARIGNANI - CiOTOLA - PACETTI - PANELla 1986, op. cit.

${ }^{20}$ WARD-PERKINS 2008, pp. 225-229

${ }^{21}$ GOFFART 1980, op. cit. Si veda anche la dettagliata analisi che compare in WARD-PERKINS 2008, op. cit.

${ }^{22}$ HeAther 2008, op. cit.; WARD-PERKINS 2008, op. cit.

${ }^{23}$ AZZARA 2003, pp. 51-86.
} 
decreto sopra menzionato ${ }^{24}$. È da dire, inoltre, che molti furono i danni provocati anche dai barbari che devastarono l'Italia nel periodo compreso dal 401 al 412 d.C.: tanti furono i problemi provocati dagli incursori che il governo centrale fu costretto a concedere le remissione dei tributi per ben cinque anni (413 - 418 d.C.). Un decreto stabilì, infatti, di condonare i quattro quinti delle imposte ma questo non bastò a sistemare la situazione economica dei contribuenti visto che nel 418 fu concessa una proroga $^{25}$. Il decreto e la relativa posticipazione dimostrano non solo che i danni provocati dai barbari furono ingenti ma anche che le invasioni costrinsero l'Impero a diminuire la base impositiva e a perdere, come si spiegherà nel corso di questo paragrafo, ingenti risorse proprio nel momento in cui si aveva più bisogno di liquidità.

I territori, inoltre, che furono presi con la forza non costituivano più delle porzioni dello stato romano ma, come si evince dalle fonti, furono strappati a Roma: la conseguenza fu la perdita dei tributi anche di quelle porzioni dell'impero in cui si insediarono i barbari nei primi decenni del V secolo d.C. (Gallia e Spagna) con il risultato che i problemi per la pars Occidentalis peggiorarono. Risulta evidente, infatti, che se Roma perse la base impositiva non poteva pagare e sostenere ne l'esercito ne, tantomeno, l'Annona civica ${ }^{26}$.

A queste problematiche si aggiunge la conquista dell'Africa da parte dei Vandali che aggrava la situazione già difficile dell'Impero: con la conquista di Cartagine nel 439, che sancisce la conquista del granaio di Roma, Genserico acquisisce anche parte della flotta romana e devia gli approvvigionamenti verso la Spagna e la Francia meridionale. Non sembra essere un caso infatti che a partire dalla conquista vandala, periodo in cui la produzione di ceramica sigillata africana aumenta a causa della riorganizzazione delle officine, aumentano le importazioni in Spagna e in Francia e iniziano a diminuire a Roma e in Italia meridionale ${ }^{27}$.

I dati materiali inoltre dimostrano che, alla fine del V secolo, ormai dopo la caduta dell'Impero romano d'Occidente, le importazioni in Italia sia di ceramica sigillata africana che di anfore africane e orientali sono di gran lunga minori rispetto a quelle di appena 50 anni prima ${ }^{28}$.

Per comprendere i motivi di questo declino bisogna, a questo punto, analizzare l'organizzazione dell'economia tardoantica e il legame che esiste tra questa e le invasioni barbariche. In questo periodo, infatti, Roma dipendeva dall'Africa sia per l'approvvigionamento del grano che per quello dell'olio: entrambi erano utilizzati sia per l'annona militare che per quella civica che, come sappiamo, erano a totale carico dello stato $^{29}$. I carichi provenienti dall'Africa, quindi, erano nella quasi totalità pagati

\footnotetext{
${ }^{24}$ L'analisi è ottimamente condotta in WARD-PERKINS 2008, op. cit.

${ }^{25}$ Per il decreto del 413 si veda il Codice Teodosiano XI.28.7. Per la proroga si veda il Codice Teodosiano XI.28.12.

${ }^{26}$ Questo argomento è stato analizzato analiticamente in PETRALÌA 1995, op. cit.

27 Lo studio compiuto da Paul Reynolds consente di individuare le conseguenze che provoca la conquista dell'Africa da parte dei Vandali. Nel periodo compreso tra il 419 e il 439 d.C. si assiste alla riorganizzazione delle officine che producevano ceramica sigillata africana che provoca l'aumento delle medesime. Questo fenomeno non è accompagnato da un accrescimento delle importazioni a Roma e in Italia meridionale: queste rimangono uguali e, dopo il 430 d.C., si assiste ad una leggera ma costante decrescita nelle quantità importate. Tale dato è completamente diverso per la Francia meridionale e per la Spagna che in questo stesso periodo vedono aumentare la quantità di ceramica acquisita dall'Africa che si realizza proprio a discapito di Roma e dell'Italia meridionale. Questo elemento permette di ipotizzare che i Vandali sono causa di un rimodellamento delle rotte che favorisce l'occidente del Mediterraneo. Sull'argomento si veda lo studio di REYNOLDS 1995, pp. 5-141. Si consulti in particolare il $\mathrm{V}$ capitolo.

${ }^{28}$ CARIGNANI - CiOTOLA - PACETTI - PANELlA 1986, op. cit.

${ }^{29}$ PANELLA 1993, op. cit.
} 
dall'Impero e su questi viaggiavano anche le vettovaglie, come la sigillata africana, di largo consumo che risultano essere trasportate a titolo gratuito poiché il trasporto era già pagato ${ }^{30}$.

Questo sistema era retto dall'imposizione fondiaria diretta che risulta essere versata da ciascun contribuente e veniva raccolta dalle curie cittadine e dirottata verso il centro. Successivamente lo stato versava lo stesso quantitativo verso le periferie attraverso la paga dei soldati e dei funzionari statali.

Il sistema che, in verità, appare abbastanza semplice ma che risulta complesso per l'estensione dell'impero, consentiva ai contribuenti di procurarsi le monete per pagare l'imposta attraverso la vendita del surplus agricolo, che confluiva nell'annona, agli organi periferici dell'amministrazione: in questo modo si mantenevano salde le finanze dello stato ${ }^{31}$.

Ne consegue, però, che non appena questo meccanismo veniva a rompersi allo Stato mancavano le risorse per pagare i trasporti e i costi di transazione diventavano insostenibili: se questi erano stati rimossi dal delicato sistema fiscale non appena la macchina si inceppava i prezzi ritornavano ad essere esorbitanti per il comune cittadino e le merci che, precedentemente erano a basso costo, ritornavano ad essere disponibili soltanto per l'elite ${ }^{32}$.

È da chiedersi, ora, in che modo i barbari abbiano alterato l'economia romana e per fare ciò bisogna comprendere quali fossero i meccanismi che ad essa soggiacevano.

È da dire che a partire dalla fine del II secolo d.C. il centro dell'impero era dipendente dalle periferie: il confronto tra l'Urbe e le province aveva visto predominare le seconde sulla prima. Questa soggezione era palese dal punto di vista economico: l'intero sistema, come si è visto, era retto da un "fragile" equilibrio che vedeva, però, la dipendenza del centro dalla periferia. L'impianto economico, infatti, si reggeva sui tributi versati dagli abitanti delle province e la riforma di Diocleziano inasprì il legame. Le province, inoltre, contribuivano alla prosperità dello Stato anche attraverso l'attività produttiva: il centro consumava ciò che veniva fabbricato nelle province $^{33}$.

Questa dipendenza, quindi, rendeva il sistema economico particolarmente fragile: non appena veniva a mancare la base impositiva e i prodotti delle province il centro soffriva. I barbari si inseriscono proprio all'interno di questa defaillance e vanno ad alterare il sistema economico attraverso diverse azioni: conquistano alcuni territori (Gallia, Spagna e Inghilterra) con la conseguente interruzione del flusso dei tributi al centro; acquisiscono l'Africa settentrionale e provocano la "rottura" dei flussi commerciali verso il centro attraverso la modifica delle rotte commerciali che vengono dirottate verso la Francia e la Spagna ${ }^{34}$.

L'alterazione del sistema, dunque, è provocato dai Barbari che costituiscono l'elemento determinante per la modifica di una struttura economica che verrà strutturata su basi diverse. Nel sottile confronto, infatti, che si realizza tra centro e periferia è la seconda ad emergere: il primo implode poiché non riesce a mutare il

\footnotetext{
${ }^{30}$ CARANDINI 1993, op. cit.

${ }^{31}$ PETRALia 1995, op. cit.

32 CARANDINI 1993, op. cit.

33 CARANDINI 1993, op. cit.

${ }^{34}$ REYNOLDS 1995, op. cit.
} 
sistema mentre la periferia emerge attraverso la generazione di un nuovo meccanismo che non è più basato sulla tassazione e sulla "redistribuzione" della ricchezza ${ }^{35}$.

I barbari, in conclusione, possono essere visti come l'elemento catalizzatore per la rottura del fragile equilibrio che si era stabilito fra centro e periferia. Questi, infatti, non possono essere visti come la causa della rottura: l'equilibrio tra i due poli si era spezzato da tempo.

La divisione dell'Impero aveva già "rotto" quell'unità economica che consentiva alla Stato di affrontare le avversità: la liquidità necessaria era disponibile anche nei periodi di crisi profonda poiché non si dipendeva da una sola ricca provincia come si verifica nell’'Impero romano d’Occidente a partire dal 395 d.C. ${ }^{36}$

Si può dire, quindi, che la divisione dell'impero in due compagini statali ruppe quel sottile equilibrio presente tra centro e periferia e rese il primo completamente dipendente dalla seconda: non appena l'assetto di quest'ultima mutava anche il centro cambiava inesorabilmente.

L'arrivo dei Barbari provoca la rottura del rapporto tra centro e periferia: emergono nuovi sistemi economici che non prevedono la tassazione gestita da un solo centro. Le invasioni sono causa di un nuovo mondo dove coesistono più centri e molteplici mondi economici che si strutturano su basi diverse. I barbari, quindi, rompono un sistema facendo emergere la periferia: il centro collassa su se stesso e il sistema economico si sfalda ${ }^{37}$.

\footnotetext{
${ }^{35}$ L'arrivo dei barbari favorisce l'introduzione di un sistema economico fondato su basi diverse: emergono le relazioni parentelari e sono le élites che si sostituiscono allo Stato centrale nel controllo dello scambio degli oggetti di lusso. Sull'argomento si veda CARANDINI 1993, op. cit.

${ }^{36}$ Prima del 395 d.C., data della divisione in due parti dell'Impero romano, Roma riesce ad affrontare molteplici crisi economiche che non portano alla distruzione di un sistema oramai consolidato da tempo. La possibilità, infatti, di potersi servire della forza produttiva e tributaria di diverse province non ne mette a rischio la struttura. Con la divisione le due parti la pars occidentalis e orientalis prendono due strade diverse: la prima risente delle invasioni poiché con la perdita dei territori perde anche la base impositiva e produttiva; la seconda non avverte, almeno sino alle conquiste degli Avari, nessuna crisi poiché riesce a mantenere molti territori e molteplici risorse.

${ }^{37}$ La mutazione del sistema economico non provoca la chiusura del mondo occidentale agli afflussi commerciali provenienti dall'Impero romano d'Oriente: alla luce dei dati archeologici sarebbe assurdo affermare questo. Ma il tutto non è più gestito dallo Stato e questo provoca due conseguenze: si innalzano i prezzi dei prodotti poiché aumenta il costo di transazione (i carichi non sono più pagati dal governo centrale per provvedere all'annona civica e militare), e le merci che prima risultavano di largo consumo poiché economiche, come la sigillata africana, diventano prodotti per l'èlite. Sull'argomento si veda CARANDINI 1993, op. cit.
} 


\section{Bibliografia}

ANSELMINO - COLETTI - FERRANTINI - PANELlA 1986

L. Anselmino, C. M. Coletti, M. L. Ferrantini, C. Panella, Ostia. Terme del nuotatore, in Società romana e impero tardo antico, Vol. III, (a cura di)

Andrea Giardina, Laterza, Roma - Bari 1986, pp. 45-81.

AZZARA 2003

C. Azzara, Le invasioni barbariche, Il Mulino, Bologna 2003, pp. 51-86.

BOWERSOCK 1996

G. Bowersock, The Vanishing Paradigm of the Fall of Rome, in Bullettin of the American Academy of Arts and Sciences 49 (1996), pp. 29-43.

BOWERSOCK 2002

G. Bowersock, Antico e tardoantico oggi, in Vera D., Antico e tardoantico oggi, RSI 114, 2002, pp. 376-379.

BOWERSOCK 2004

G. BOWERSOCK, Riflessioni sulla periodizzazione dopo «Esplosione di tardoantico» di Andrea Giardina, in Gli Spazi del tardoantico, Studi Storici, 45, 2004, Carocci, Roma, pp. 7-13 .

BROWN 1974

P. Brown, Il mondo tardoantico. Da Marco Aurelio a Maometto, Einaudi, Torino 1974.

BROWN 2001

P. Brown, Genesi della tardo antichità, Einaudi, Torino 2001.

CARANDINI 1986

A. CARANDini, Il Mondo della tarda antichità visto attraverso le merci, in Società romana e impero tardo antico, Vol. III, (a cura di) Andrea Giardina, Laterza, Roma - Bari 1986, pp. 9-19.

CARANDINI 1993

A. CARANDini, L'ultima civiltà sepolta o del massimo oggetto desueto, secondo un archeologo, in Storia di Roma, Vol. III, tomo 2, a cura di Carandini A., Cracco Ruggini L., Giardina A., Einaudi, Torino 1993, pp. 1138.

CARIGNANI - CIOTOLA - PACETTI - PANELLA 1986

A. Carignani, A. Ciotola, F. Pacetti, C. Panella, Roma. Il contesto del tempio della Magna Mater sul Palatino, in Società romana e impero tardo antico, Vol. III, (a cura di) Andrea Giardina, Laterza, Roma - Bari 1986, pp. 27-43.

CIPRIANO - VOLPE 1986

M. T. Cipriano, G. VolPe, Luni (Etruria). Il contesto del centro urbano, in Società romana e impero tardo antico, Vol. III, (a cura di) Andrea Giardina, Laterza, Roma - Bari 1986, pp. 89-96.

COURCELLE 1948

P. COURCELlE, Histoire littéraire des grandes invasions germaniques, Hachette, Paris 1948, pp. 58-79.

COURTOIS 1955

C. Courtois, Les Vandales et l'Afrique, Arts et Métiers graphiques, Paris 1955, pp. 42-51. 


\section{CRACCO RUgGini 1993}

L. CRAcCo Ruggini, Il Tardoantico: per una tipologia dei punti critici, in Storia di Roma, Vol. III, tomo 1, a cura di Carandini A., Cracco Ruggini L., Giardina A., Einaudi, Torino 1993, pp. XXXIII-XXXVI sgg.

Cracco Ruggini 2002

L. Cracco Ruggini, Antico e tardoantico oggi, in Vera D., Antico e tardoantico oggi, RSI, 114, 2002, pp. 351-379.

Cracco Ruggini 2004

L. CRACCO Ruggini, Come e perché è «esploso» il tardoantico oggi, in Gli spazi del tardoantico, Studi Storici, 45, 2004, Carocci, Roma, pp. 15-23.

DE GIOVANNi 2007

L. De Giovanni, Istituzioni. Scienza giuridica. Codici nel mondo tardoantico. Alle radici di una nuova storia, L’Erma di Bretschneider, Roma 2007, pp. 138

DEMANDT 2007

A. Demandt, Die Spätantike. Römische Geschichte von Diocletian bis Justinian (284-565 n. Chr.). München (Handbuch der Altertumswissenschaft 3, 6) 22007, pp. 578-608.

GIARDINA 1999

A. GiardinA, Esplosione di tardoantico, in Studi Storici, 40, 1999, Carocci, Roma, pp. 157-180.

GOFFART 1980

W. Goffart, Barbarians and Romans. The Techniques of Accomodation, Princeton University Press, Princeton 1980.

HEATHER 2008

P. HeAther, La caduta dell'impero romano. Una nuova storia, Garzanti, Milano 2008.

MARCONE 2004

A. MARCONE, La tarda antichità o delle difficoltà delle periodizzazioni, in Gli spazi del tardoantico, Studi Storici, 45, Carocci, Roma 2004, pp. 25-36.

MARCONE 2007

A. Marcone, La caduta di Roma all'inizio del III Millennio, in Antidoron. Studi in onore di Barbara Scardigli Forster, a cura di P. Desideri, M. Poggi, M. Pani, Edizioni ETS, Pisa 2007, pp. 267-280.

MATTHEWS 1975

J. MATTHEWS, Western Aristocracies and Imperial Court AD 364-425, Clarendon Paperbacks, Oxford 1975.

PANELLA 1986

C. Panella, Per lo studio dei contesti e delle merci tardoantiche, in Società romana e impero tardo antico, Vol. III, (a cura di) Andrea Giardina, Laterza, Roma - Bari 1986, pp. 21-23.

PANELLA 1993

C. Panella, Merci e scambi nel Mediterraneo tardoantico, in Storia di Roma, Vol. III, tomo 2, a cura di Carandini A., Cracco Ruggini L., Giardina A., Einaudi, Torino 1993, pp. 613-697.

PETRALÌ̀ 1995

G. Petralìa, A proposito dell'immortalità di «Maometto e Carlomagno (o di Costantino), in Storica I, Donzelli, Roma 1995, pp. 38-87. 
Frankfurter elektronische Rundschau zur Altertumskunde 16 (2011)

PIRENNE 1937

H. Pirenne, Mahomet e Charlemagne, Nouvelle Société d'éditions, ParisBruxelles 1937.

REYNOLDS 1995

P. REYNOLDS, Trade in the Western Mediterranean AD 400-700: The Ceramic evidence, BAR International Series 604, 1995, pp. 5-141.

SCHIAVONE 1996

A. SCHIAVOne, La storia spezzata. Roma antica e occidente moderno, Laterza, Roma-Bari 1996.

VERA 2002

D. VERA, Antico e tardoantico oggi, RSI, 114, 2002, pp. 349-350.

WARD - PERKINS 2008

B. WARD - PERKINS, La caduta di Roma e la fine della civiltà, Laterza, RomaBari 2008.

\section{Kontakt zum Autor:}

Dott. Alberto Giudice

Professore di Archeologia Cristiana

Pontificia Facoltà Teologica dell’Italia Meridionale

Istituto Superiore di Scienze Religiose - Capua

Via Pozzo San Giovanni 1

I-84030 Caselle in Pittari (SA)

E-Mail: alberto.giudice@yahoo.de 